\title{
Cross sectional STEM imaging and analysis of multilayered two dimensional crystal heterostructure devices
}

\author{
Sarah J. Haigh ${ }^{1}$, Aidan P. Rooney ${ }^{1}$, Eric Prestat ${ }^{1}$, Fred. Withers ${ }^{2}$, O. Del Pozo Zamudio ${ }^{3}$, Artem
} Mishchenko $^{2}$, Ali. Gholinia ${ }^{1}$, K. Watanabe ${ }^{4}$, T. Taniguchi ${ }^{4}$, A. I. Tartakovskii ${ }^{3}$, Andre K. Geim ${ }^{5}$, Konstantin. S. Novoselov ${ }^{2}$,

1. School of Materials, University of Manchester, Manchester, M13 9PL, UK

2. School of Physics and Astronomy, University of Manchester, Manchester, M13 9PL, UK

3. Department of Physics and Astronomy, University of Sheffield, Sheffield, S3 7RH, UK

4. National Institute for Materials Science, 1-1 Namiki, Tsukuba 305-0044, Japan.

${ }^{5}$ Manchester Centre for Mesoscience and Nanotechnology, University of Manchester, Manchester, M13 9PL, UK

The number of two dimensional crystals successfully isolated has expanded rapidly in recent years, providing a wide variety of interesting electronic properties.[1] By combining different two dimensional crystals within a Van der Waals heterostructure stack it is possible to produce devices with bespoke electronic bandstructure.[1] This is fast establishing a new generation of optical and electronic devices with advanced functionality.[2-6]

Our Van der Waals heterostructures have been synthesized by sequential layering of different twodimensional crystals to form a stack on a silicon wafer substrate.[1] The crystals have been produced by mechanical exfoliation and transferred by deposition and subsequent removal of the polymeric support layers. Despite the inherently 'dirty' nature of this processing we have found that the heterostructure stacks form large areas of pristine synthetic crystal, free from hydrocarbon contaminants at the atomic scale.[7] This has exciting implications for device performance and design.

For example, the device shown in Figure 1 is a light-emitting diode (LED) composed of multiple quantum wells separated by h-BN tunneling layers.[8] Graphene electrodes above and below the stack allow charge carrier pairs to tunnel through the insulating h-BN layers into the $\mathrm{MoS}_{2}$ quantum wells, where they form excitons before undergoing radiative recombination, measured as light emission. Optical and electronic characterisation has shown electroluminescence quantum efficiencies of up to 5\% are possible for these multiple quantum well devices, comparable with modern organic LEDs. Device performance depends critically on the width of the h-BN tunneling barriers as well as the thickness and uniformity of $\mathrm{MoS}_{2}$ layers within the device. These parameters are difficult or impossible to characterize within such a complex multilayer device. The only method by which this atomic scale structural detail can be obtained is cross sectional scanning transmission electron microscope (STEM) imaging and analysis.

After the device has undergone electronic testing, a site specific cross section can be extracted from the active area of the device using the focused ion beam 'lift out' method. STEM imaging of the device cross section reveals that it contains four atomically flat $\mathrm{MoS}_{2}$ monolayers. The composition of sequential device layers can be identified in elemental maps extracted from energy dispersive x-ray spectrum image data (Figure $1 \mathrm{c}$ ). The number of h-BN layers separating each $\mathrm{MoS}_{2}$ monolayer can be determined and the location of the top graphene electrode identified (indicated by dashed white line in Figure $1 \mathrm{c}$ iii). 
We have used a similar cross sectional STEM imaging and analysis approach to investigate a range of graphene - transition metal dichalcogenides (TMDCs) heterostructures at the atomic scale. We demonstrate that this technique can provide unprecedented insights into the structural nature and failure mechanisms of these complex devices.[4, 6-8]

\section{References}

[1] Geim, A.K. and I.V. Grigorieva,. Nature, 2013. 499(7459): p. 419-425.

[2] Britnell, L., et al. Science, 2012. 335(6071): p. 947-950.

[3] Britnell, L., et al. Science, 2013. 340(6138): p. 1311-1314.

[4] Georgiou, T., et al. Nat Nano, 2013. 8(2): p. 100-103.

[5] Yu, W.J., et al. Nat Nano, 2013. 8(12): p. 952-958.

[6]Withers, F. et al. Nano Letters, 2014. 14(7): p. 3987-3992.

[7]Haigh, S.J. et al. Nat Mater, 2012. 11(9): p. 764-767.

[8] Withers, F. et al. Nat Mater, 2015. advance online publication.

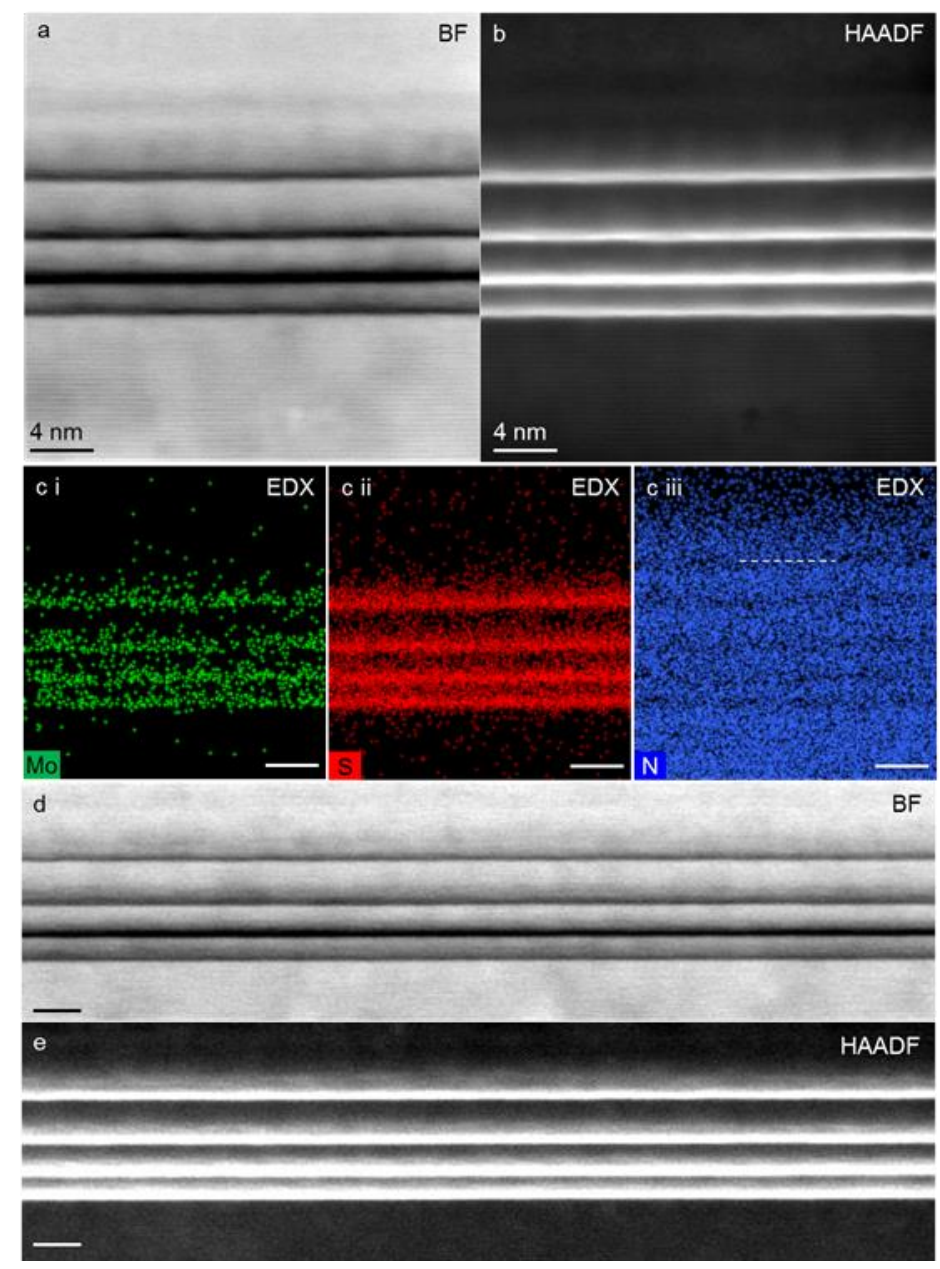

Figure 1 Cross sectional Imaging of $\mathrm{MoS}_{2}$ multilayer quantum well. (a) Bright field and (b) high angle annular dark field STEM images of the four layer MoS2 heterostructure cross-section. Boron nitride lattice fringes are clearly visible in both images, as are the position of the $\mathrm{MoS}_{2}$ monolayers. (c i-iii) Show elemental maps for Mo, $\mathrm{S}$ and $\mathrm{N}$ extracted from energy dispersive $\mathrm{X}$ ray (EDX) spectrum image data. The top graphene electrode can be seen as a deficiency in the nitrogen EDX map (indicated by dashed white line in (ciii)). (d) and (e) show bright field and high angle annular dark field images at lower magnification. All scale bars are $4 \mathrm{~nm}$. 